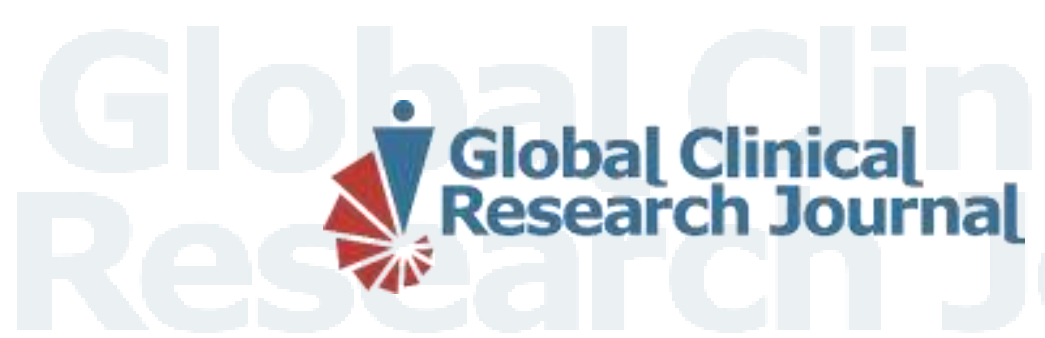

\title{
Aleitamento materno e a pandemia da COVID-19
}

Breastfeeding and the COVID-19 pandemic

Lactancia materna y la pandemia de COVID-19

\author{
Ana Luíza de Siqueira Simão \\ ORCID: 0000-0001-9043-3421 \\ Beatriz de Oliveira Chouzende ${ }^{1}$ \\ ORCID: 0000-0001-9783-5861 \\ Kawany de Oliveira Rodrigues Duarte ${ }^{1}$ \\ ORCID: 0000-0003-4962-6082
}

\author{
Samara Gomes Rodrigues ${ }^{1 *}$ \\ ORCID: 0000-0001-5855-6130 \\ Luciane Andrea Aver ${ }^{2}$ \\ ORCID: 0000-0002-2432-7628 \\ Marcia Carneiro Saco ${ }^{1}$ \\ ORCID: 0000-0002-3705-9079
}

\footnotetext{
${ }^{1}$ Centro Universitário São Camilo. São Paulo, Brasil.

${ }^{2}$ Universidade Federal de São Paulo. São Paulo, Brasil.

*Autor correspondente: E-mail: samaragomesrodrigues26@gmail.com
}

\section{Resumo}

Objetivou-se nesse estudo compreender os impactos do SARS-CoV-2 no aleitamento materno. Trata-se de uma revisão integrativa da literatura realizada nas bases de dados online LILACS, BDENF e MEDLINE. Os descritores utilizados na busca foram "Aleitamento materno" AND "Coronavírus" AND "Período pós-parto". A busca compreendeu o período janeiro 2021. Foram selecionados estudos com idiomas português e inglês. Onze artigos atenderam aos critérios de elegibilidade e compuseram a leitura da síntese da presente revisão. De acordo com os estudos existem diversos fatores que impactam no aleitamento materno frente ao SARSCoV-2, podemos citar que a insegurança das lactantes sendo o maior fator que impede a manutenção da amamentação e as incertezas que ao longo da pandemia cercam a mesma. Conclui-se que o aleitamento materno de mães com COVID-19 é seguro, seguindo as medidas de controle de infecção adequadas para evitar o contágio mãe-bebê, e para diminuir o impacto do desmame precoce devemos realizar atividades educativas no período pré-natal e realizar treinamento com a equipe multiprofissional para incentivar o aleitamento exclusivo frente aos seus benefícios.

Descritores: Aleitamento Materno; Coronavírus; Período Pós-Parto; Leite Humano; Lactação; Imunização Passiva.

Como citar este artigo:

Simão ALS, Chouzende BO, Duarte KOR, Rodrigues SG, Aver LA, Saco MC. Aleitamento materno e a pandemia da COVID-19. Glob Clin Res. 2021;1(1):e6.

Editor Chefe: Caroliny dos Santos Guimarães da Fonseca

Editor Executivo: Kátia dos Santos Armada de Oliveira 
The aim of this study was to understand the impacts of SARS-CoV-2 on breastfeeding. This is an integrative literature review carried out in the online databases LILACS, BDENF and MEDLINE. The descriptors used in the search were "Breastfeeding" AND "Coronavirus" AND "Postpartum period". The search covered the period January 2021. Studies in Portuguese and English were selected. Eleven articles met the eligibility criteria and composed the reading of the summary of this review. According to the studies, there are several factors that impact breastfeeding in the face of SARS-CoV-2, we can mention that the insecurity of lactating women is the biggest factor that prevents the maintenance of breastfeeding and the uncertainties that surround it throughout the pandemic. It is concluded that breastfeeding of mothers with COVID-19 is safe, following the appropriate infection control measures to prevent mother-infant contagion, and to reduce the impact of early weaning, we must carry out educational activities in the prenatal period and carry out training with the multidisciplinary team to encourage exclusive breastfeeding in view of its benefits.

Descriptors: Breastfeeding; Coronavirus; Postpartum; Human Milk; Lactation; Passive Immunization.

\section{Resumén}

El objetivo de este estudio fue comprender los impactos del SARS-CoV-2 en la lactancia. Se trata de una revisión integradora de la literatura realizada en las bases de datos en línea LILACS, BDENF y MEDLINE. Los descriptores utilizados en la búsqueda fueron "Lactancia materna" Y "Coronavirus" Y "Período posparto". La búsqueda cubrió el período de enero de 2021. Se seleccionaron estudios en portugués e inglés. Once artículos cumplieron con los criterios de elegibilidad y compusieron la lectura del resumen de esta revisión. Según los estudios, son varios los factores que impactan la lactancia materna ante el SARS-CoV-2, podemos mencionar que la inseguridad de las mujeres lactantes es el mayor factor que impide el mantenimiento de la lactancia materna y las incertidumbres que la rodean a lo largo de la pandemia. Se concluye que la lactancia materna de las madres con COVID-19 es segura, siguiendo las adecuadas medidas de control de infecciones para prevenir el contagio madre-lactante, y para reducir el impacto del destete precoz, debemos realizar actividades educativas en el período prenatal y realizar capacitaciones con el equipo multidisciplinario para fomentar la lactancia materna exclusiva en vista de sus beneficios.

Descriptores: Amamantamiento; Coronavirus; Período Posparto; Leche Humana; Lactancia; Inmunización Pasiva.

\section{Introdução}

O aleitamento materno (AM) além de ser considerado a mais eficiente maneira de estabelecer vínculo entre mãe e filho, é o processo pelo qual o lactente receberá o seu alimento ${ }^{1}$. O leite materno é o alimento mais completo para o lactente, beneficiando-o com todos os nutrientes necessários para que ele se desenvolva e cresça de forma

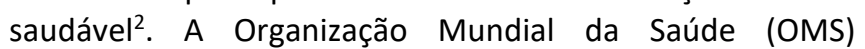
recomenda o aleitamento materno até os 2 anos de idade, e de forma exclusiva até os 6 meses $^{3}$.

É definido o AM quando a criança recebe leite materno independente de receber outros alimentos ou não, já o aleitamento materno exclusivo (AME) é definido quando a criança é alimentada apenas com leite humano, podendo ser este direto da mama ou ordenhado, não havendo exceções para alimentos sólidos e líquidos. Também existe a definição de aleitamento materno complementado (AMC) na qual a criança recebe além do leite materno outros alimentos podendo ser estes sólidos, semissólidos ou líquidos. É importante enfatizar que o leite materno possui o equilíbrio adequado de nutrientes que são fornecidos em uma forma biodisponível e facilmente digerível, a genética e a nutrição materna influenciam na sua composição, além disso com o decorrer do tempo ele muda de acordo com as necessidades do lactente para garantir a sua nutrição e desenvolvimento ${ }^{1}$.

Recentemente, em dezembro de 2019, ocorreu a transmissão de um novo vírus chamado de coronavírus denominado SARS-CoV-2 identificado em Wuhan na China, causador da doença COVID-19. Logo, esta doença começou a ser disseminada e transmitida de pessoa para pessoa. A COVID-19 apresenta um quadro clínico que varia de infecções assintomáticas a quadros graves, podendo apresentar quadro respiratório agudo e necessidade de atendimento hospitalar ${ }^{4}$.

Os sintomas mais comuns são tosse, febre, coriza, dor de garganta, dificuldade para respirar, perda de olfato, alteração do paladar, distúrbios gastrintestinais (náuseas/vômitos/diarreia), cansaço, diminuição do apetite e dispneia. Idosos e pessoas com comorbidades crônicas são as que mais apresentam complicações ${ }^{4}$.

O número de infectados por coronavírus no mundo é impactante. Foram confirmados pouco mais de 86 milhões de casos de COVID-19 e 1.890 .342 mortes até o início de janeiro de 2021. Apesar dos avanços tecnológicos para compreender esta doença e suas interações com o organismo humano, trata-se de algo recente, e faz-se 
necessário a busca por diferentes dados que possam agregar conteúdo sobre COVID-195.

A transmissão e fisiopatologia do SARS-CoV-2 são gradualmente conhecidas entre várias populações, mas os efeitos da COVID-19 na saúde pública nas mulheres e seus resultados não devem ser ignorados. Em mulheres grávidas e perinatais, a transmissão vertical de SARS-CoV-2 de uma mãe infectada para seu recém-nascido é uma questão controversa. Segundo dados das Secretarias Estaduais de Saúde, até 19 de novembro, o Brasil registrava 12.104 casos de COVID-19 em gestantes e 223 mortes pela doença ${ }^{6}$.

A Organização Mundial da Saúde (OMS) orienta a manutenção da amamentação por falta de elementos que comprovem que o leite materno possa disseminar o coronavírus, até o momento desta publicação. Objetivou-se nesse estudo compreender os impactos do SARS-CoV-2 no aleitamento materno.

\section{Metodologia}

Trata-se de uma revisão integrativa, cujo modalidade de estudo tem importância para tomada de decisão frente a conduta com profissionais de saúde consiste na construção de uma análise ampla da literatura, que contribui para a síntese de um determinado assunto, esse método de pesquisa baseia-se em estudos anteriores ${ }^{7}$, com
Aleitamento materno e a pandemia da COVID-19 Simão ALS, Chouzende BO, Duarte KOR, Rodrigues SG, Aver LA, Saco MC as seguintes etapas: formulação da questão norteadora, coleta de estudos/artigos, análise e interpretação, finalizando com apresentação dos resultados.

A busca foi realizada no período de janeiro de 2021, por meio dos descritores "Aleitamento materno", "Coronavírus" e "Período pós-parto" combinados entre si e com o uso do recurso booleano $A N D$, na Biblioteca Virtual em Saúde (BVS), acessando as bases de dados: Literatura Latino-Americana e do Caribe em Ciências da Saúde (LILACS), Banco de Dados em Enfermagem (BDENF), MEDLINE, nos últimos 5 anos de publicação.

Permitiu a obtenção de um total de 108 artigos, sendo 89 desses artigos encontrados na base de dados MEDLINE, 15 estudos na LILACS e 4 na BDENF. 98 estudos disponíveis na íntegra que apresentavam relação com os critérios de inclusão: artigos no idioma português e inglês, publicados em periódicos nacionais e internacionais, e que respondem à questão norteadora. Foram excluídos 12 artigos por serem repetidos, 40 trabalhos de revisão integrativa e 35 não responderam à seguinte questão norteadora: "Quais os impactos do SARS-CoV-2 no aleitamento materno".

A seleção de artigos para composição da presente revisão integrativa foi organizada na Figura 1.

Figura 1. Estratégias de busca utilizadas nas bases de dados. São Paulo, SP, Brasil, 2021

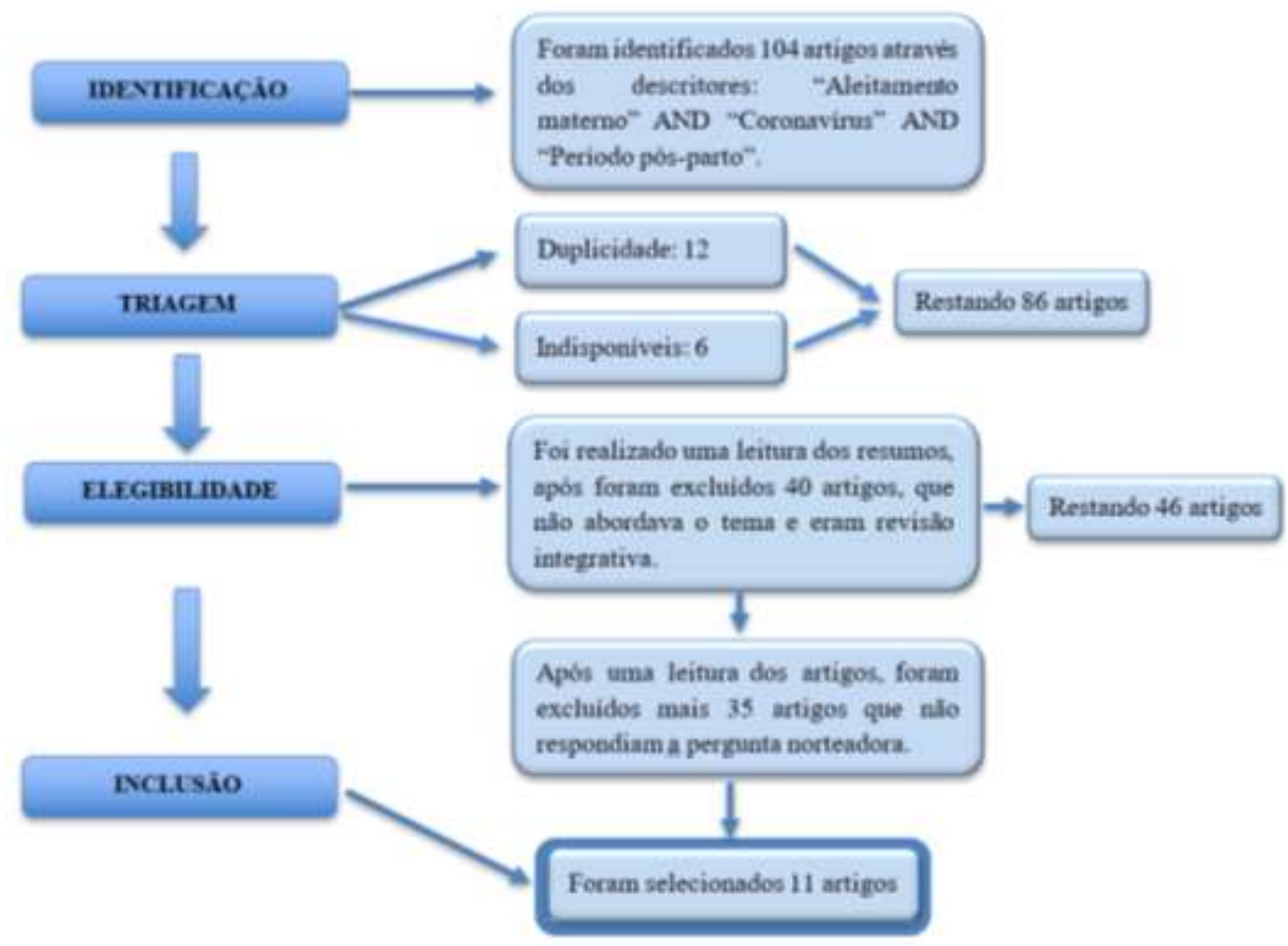

Conforme apontam lacunas do conhecimento que precisam ser preenchidas com a realização de novos estudos, em fontes governamentais, após a leitura dos títulos, dos resumos e da análise dos artigos, permitiu a seleção de 11 estudos.

\section{Resultados}

Os 11 artigos selecionados foram organizados no Quadro 1 e 2, onde identifica-se a amostra dos estudos selecionados para a presente revisão integrativa segundo ano de publicação, autores, título e os objetivos da pesquisa (Quadro 1). 
Aleitamento materno e a pandemia da COVID-19

Simão ALS, Chouzende BO, Duarte KOR, Rodrigues SG, Aver LA, Saco MC

Quadro 1. Quadro sinóptico dos artigos selecionados contendo ano de publicação, autores, título e resultados. São Paulo, SP, Brasil, 2021

\begin{tabular}{|c|c|c|c|}
\hline ANO DE PUBLICAÇÃO & AUTORES & TíTULO & OBJETIVOS \\
\hline Janeiro/2021 & $\begin{array}{l}\text { Brown, Amy; } \\
\text { Shenker, Natalie. }\end{array}$ & $\begin{array}{l}\text { Experiences of breastfeeding } \\
\text { during COVID-19: Lessons for } \\
\text { future practical and emotional } \\
\text { support. }\end{array}$ & $\begin{array}{l}\text { Analisar as experiências de mais de } 1200 \text { mulheres com } \\
\text { bebês menores de } 1 \text { ano para entender como a pandemia } \\
\text { de COVID-19 afetou suas atitudes, escolhas e resultados } \\
\text { de alimentação infantil. }\end{array}$ \\
\hline Outubro/2020 & $\begin{array}{l}\text { Marchiori, } \\
\text { Giovanna Rosario } \\
\text { Soanno; et al. }\end{array}$ & $\begin{array}{l}\text { Ações da enfermagem nos bancos } \\
\text { de leite humano em tempos de } \\
\text { COVID-19. }\end{array}$ & $\begin{array}{l}\text { Analisar as ações das coordenações dos Bancos de Leite } \\
\text { Humano para favorecer a continuidade da amamentação } \\
\text { na pandemia da COVID-19. }\end{array}$ \\
\hline Novembro/2020 & $\begin{array}{l}\text { Lebrão, Cibele } \\
\text { Wolf; et al. }\end{array}$ & $\begin{array}{l}\text { Early Identification of IgA Anti- } \\
\text { SARSCoV-2 in Milk of Mother With } \\
\text { COVID-19 Infection. }\end{array}$ & $\begin{array}{l}\text { Identificar IgA anti-SARS-CoV-2 no leite materno com } \\
\text { infecção por COVID-19. }\end{array}$ \\
\hline Agosto/2020 & $\begin{array}{l}\text { Ceulemans, } \\
\text { Michael; et al. }\end{array}$ & $\begin{array}{l}\text { SARS-CoV-2 Infections and Impact } \\
\text { of the COVID-19 Pandemic in } \\
\text { Pregnancy and Breastfeeding: } \\
\text { Results from an Observational } \\
\text { Study in Primary Care in Belgium. }\end{array}$ & $\begin{array}{l}\text { Fornecer estimativas de infecções por SARS-CoV-2 entre } \\
\text { mulheres grávidas e lactantes, bem como avaliar o } \\
\text { impacto percebido pelas mulheres da pandemia em suas } \\
\text { práticas de amamentação, aconselhamento médico e } \\
\text { apoio social durante a gravidez e lactação. }\end{array}$ \\
\hline Agosto/2020 & $\begin{array}{l}\text { Pereira, Augusto; } \\
\text { et al. }\end{array}$ & $\begin{array}{l}\text { Breastfeeding mothers with } \\
\text { COVID-19 infection: a case series. }\end{array}$ & $\begin{array}{l}\text { Apresentar uma série de casos representativos de bebês } \\
\text { que receberam aleitamento materno de mães COVID-19 e } \\
\text { descrever o manejo das mães e bebês durante a } \\
\text { amamentação, a indicação da amamentação dependendo } \\
\text { da gravidade dos sintomas e a amamentação em neonatos } \\
\text { prematuros durante a admissão na UTIN. }\end{array}$ \\
\hline Agosto/2020 & $\begin{array}{l}\text { Yu, Yuanyuan; et } \\
\text { al. }\end{array}$ & $\begin{array}{l}\text { Breastfed 13-month-old infant of } \\
\text { a mother with COVID-19 } \\
\text { pneumonia: a case report. }\end{array}$ & $\begin{array}{l}\text { Documentar pela primeira vez um caso de amamentação } \\
\text { de um bebê por uma mãe infectada com SARS-CoV-2 e } \\
\text { descrever a apresentação clínica, diagnóstico, tratamento } \\
\text { e resultado. }\end{array}$ \\
\hline Agosto/2020 & $\begin{array}{l}\text { Gabriel, Miguel } \\
\text { Ángel Marín; et al. }\end{array}$ & $\begin{array}{l}\text { Negative } \text { Transmission of SARS- } \\
\text { CoV-2 to Hand-Expressed } \\
\text { Colostrum from SARS-CoV-2- } \\
\text { Positive Mothers. }\end{array}$ & $\begin{array}{l}\text { Determinar se as mães SARS-CoV-2-positivas transmitem } \\
\text { o vírus para o colostro extraído à mão. }\end{array}$ \\
\hline Agosto/2020 & $\mathrm{Wu}, \mathrm{Y}$; et al. & $\begin{array}{l}\text { Coronavirus disease } 2019 \text { among } \\
\text { pregnant Chinese women: case } \\
\text { series data on the safety of } \\
\text { vaginal birth and breastfeeding. }\end{array}$ & $\begin{array}{l}\text { Avaliar se as secreções vaginais e o leite materno de } \\
\text { mulheres com doença coronavírus } 2019 \text { (COVID - 19) } \\
\text { contêm síndrome respiratória aguda grave coronavírus } 2 \\
\text { (SARS-CoV-2). }\end{array}$ \\
\hline Julho/2020 & $\begin{array}{l}\text { Pereira, Augusto; } \\
\text { et al. }\end{array}$ & $\begin{array}{l}\text { Clinical course of coronavirus } \\
\text { disease-2019 in pregnancy. }\end{array}$ & $\begin{array}{l}\text { Descrever a nossa experiência no tratamento clínico de } 60 \\
\text { mulheres grávidas COVID - } 19 \text { - } 19 \text { positivas atendidas no } \\
\text { nosso hospital durante o primeiro mês da epidemia na } \\
\text { Espanha. }\end{array}$ \\
\hline Abril/2020 & $\begin{array}{l}\text { Ministério } \quad \text { da } \\
\text { Saúde. }\end{array}$ & $\begin{array}{l}\text { Coronavírus } \quad \text { Covid-19: } \\
\text { Orientações sobre amamentação. }\end{array}$ & $\begin{array}{l}\text { Avaliar medida para o enfrentamento da situação de } \\
\text { emergência em saúde pública no contexto da infecção da } \\
\text { COVID-19 causada pelo novo coronavírus (SARS-CoV-2), } \\
\text { sobre questionamento da Rede de Atenção à Saúde (RAS) } \\
\text { respectivo à preservação da amamentação em situação de } \\
\text { risco iminente de transmissão do respectivo vírus, em } \\
\text { situações que a mãe apresente sintomatologia compatível } \\
\text { com síndrome gripal. }\end{array}$ \\
\hline Agosto/2020 & $\begin{array}{l}\text { Lima, Ana Carolina } \\
\text { Maria Araújo } \\
\text { Chagas Costa; et } \\
\text { al. }\end{array}$ & $\begin{array}{l}\text { Consultoria em amamentação } \\
\text { durante a pandemia COVID-19: } \\
\text { relato de experiência. }\end{array}$ & $\begin{array}{l}\text { Relatar a experiência de consultoras em amamentação no } \\
\text { atendimento às lactantes durante a pandemia de COVID- } \\
19 .\end{array}$ \\
\hline
\end{tabular}

O Quadro 2, permite uma amostra dos estudos selecionados a partir da análise do título do estudo e os resultados obtidos pelos autores. 


\begin{tabular}{|c|c|}
\hline TÍTULO & RESULTADOS \\
\hline $\begin{array}{l}\text { Experiences of breastfeeding during } \\
\text { COVID-19: Lessons for future practical and } \\
\text { emotional support. }\end{array}$ & $\begin{array}{l}41,8 \% \text { das mães sentiram que a amamentação estava protegida devido ao bloqueio, mas } 27,0 \% \\
\text { das mães lutaram para obter apoio e tiveram várias barreiras decorrentes do bloqueio com } \\
\text { algumas pararam de amamentar antes de estarem prontas. Mães com menor escolaridade, com } \\
\text { condições de vida mais desafiadoras e de origem étnica negra e de minorias eram mais propensas } \\
\text { a considerar o impacto do bloqueio como um desafio e interromper a amamentação. }\end{array}$ \\
\hline $\begin{array}{l}\text { Ações da enfermagem nos bancos de leite } \\
\text { humano em tempos de COVID-19. }\end{array}$ & $\begin{array}{l}\text { Foram produzidas duas categorias: As ações de estratégias da manutenção dos serviços do Bancos } \\
\text { de Leite Humano por meios digitais como forma de garantir o distanciamento social e a } \\
\text { amamentação, em tempos de pandemia do novo coronavírus; e as ações de promoção, proteção } \\
\text { e apoio nas estratégias de manutenção da amamentação durante a pandemia. }\end{array}$ \\
\hline $\begin{array}{l}\text { Early Identification of IgA Anti-SARSCoV-2 } \\
\text { in Milk of Mother With COVID-19 Infection. }\end{array}$ & $\begin{array}{l}\text { Foi detectada imunoglobulina A anti-SARS-CoV-2 nas duas amostras avaliadas, cujos valores foram } \\
2,5 \text { e } 1,9 \text {, respectivamente. Nenhuma imunoglobulina } G \text { anti-SARSCoV- } 2 \text { foi detectada. A criança } \\
\text { amamentada exclusivamente permaneceu bem até os } 45 \text { dias de idade. }\end{array}$ \\
\hline $\begin{array}{l}\text { SARS-CoV-2 Infections and Impact of the } \\
\text { COVID-19 Pandemic in Pregnancy and } \\
\text { Breastfeeding: Results from an } \\
\text { Observational Study in Primary Care in } \\
\text { Belgium. }\end{array}$ & $\begin{array}{l}\text { Mais de } 90 \% \text { refutaram que a pandemia afetou suas práticas de amamentação, nem indicou que } \\
\text { o coronavírus era responsável pela amamentação cessação. Metade das mulheres chegou a } \\
\text { pensar em dar mais leite materno por causa do coronavírus. Em contraste, o aconselhamento } \\
\text { médico e o apoio social das mulheres foram afetados negativamente pelo bloqueio. }\end{array}$ \\
\hline $\begin{array}{l}\text { Breastfeeding mothers with COVID-19 } \\
\text { infection: a case series. }\end{array}$ & $\begin{array}{l}\text { Das } 22 \text { mães, } 20 \text { (90,9\%) optaram por amamentar seus bebês durante a internação. Iniciação } \\
\text { oportuna e o contato pele a pele na sala de parto foi realizado em } 54,5 \text { e } 59,1 \% \text {, respectivamente. } \\
\text { Oitenta e dois por cento de recém-nascidos de mães com COVID-19 foram alimentados com leite } \\
\text { materno após } 1 \text { mês, diminuindo para } 77 \% \text { em } 1,8 \text { meses. Seis de } 22(37,5 \%) \text { mães com COVID-19 } \\
\text { necessitaram de alimentação complementar transitória até que a amamentação exclusiva fosse } \\
\text { alcançada. }\end{array}$ \\
\hline $\begin{array}{l}\text { Breastfed 13-month-old infant of a mother } \\
\text { with COVID-19 pneumonia: a case report. }\end{array}$ & $\begin{array}{l}\text { Anticorpos IgM e IgG contra SARS-CoV-2 foram avaliados no soro materno e leite materno e no } \\
\text { soro infantil. O ácido nucleico SARS-CoV-2 não foram detectados no leite materno e anticorpos } \\
\text { contra SARS-CoV-2 foram detectados no soro e no leite materno. }\end{array}$ \\
\hline $\begin{array}{l}\text { Negative Transmission of SARS-CoV-2 to } \\
\text { Hand-Expressed Colostrum from SARS- } \\
\text { CoV-2-Positive Mothers. }\end{array}$ & $\begin{array}{l}\text { Obtivemos sete amostras de colostro de diferentes mães nas primeiras horas pós-parto. SARS- } \\
\text { CoV-2 não foi detectado em nenhuma das amostras de colostro obtidas em nosso estudo. }\end{array}$ \\
\hline $\begin{array}{l}\text { Coronavirus disease } 2019 \text { among pregnant } \\
\text { Chinese women: case series data on the } \\
\text { safety of vaginal birth and breastfeeding. }\end{array}$ & $\begin{array}{l}\text { Entre esses cinco partos, os resultados perinatais adversos primários incluíram parto prematuro } \\
(\mathrm{n}=2 \text { ) e pneumonia neonatal }(n=2) \text {. Uma das nove amostras de fezes foi positiva; todas as } 13 \\
\text { amostras de secreção vaginal e cinco esfregaços de garganta e quatro esfregaços anais coletados } \\
\text { de neonatos foram negativos para o novo coronavírus. No entanto, uma das três amostras de leite } \\
\text { materno foi positiva no teste de ácido nucleico viral. }\end{array}$ \\
\hline $\begin{array}{l}\text { Clinical course of coronavirus disease-2019 } \\
\text { in pregnancy. }\end{array}$ & $\begin{array}{l}\text { Níveis elevados de PCR e dímero } \mathrm{D} \text { foram os parâmetros mais frequentemente associados à } \\
\text { pneumonia grave. A razão: neutrófilos / linfócitos, foi considerada o marcador mais sensível para } \\
\text { a melhora da doença (risco relativo } 6,65 \text {; IC } 95 \% 4,1-5,9 \text { ). Durante o período do estudo, } 18 \text { das } \\
\text { mulheres ( } 78 \% \text { ) tiveram parto normal. Todos os recém-nascidos tiveram resultados negativos para } \\
\text { SARS-CoV-2 e nenhum deles foi infectado durante a amamentação. Nenhum SARS-CoV-2 foi } \\
\text { detectado no tecido placentário. }\end{array}$ \\
\hline $\begin{array}{l}\text { Coronavírus Covid-19: Orientações sobre } \\
\text { amamentação. }\end{array}$ & $\begin{array}{l}\text { Não há recomendação para a suspensão do aleitamento materno na transmissão de outros vírus } \\
\text { respiratórios }{ }^{2} \text {, recomenda que a amamentação seja mantida em caso de infecção pela SARS-CoV- } \\
2 \text {, desde que a mãe deseje amamentar e esteja em condições clínicas adequadas para fazê-lo. }\end{array}$ \\
\hline $\begin{array}{l}\text { Consultoria em amamentação durante a } \\
\text { pandemia COVID-19: relato de } \\
\text { experiência. }\end{array}$ & $\begin{array}{l}\text { Diante de sentimentos de medo e ansiedade das lactantes, as consultoras prestaram apoio por } \\
\text { meio de aconselhamento, bem como orientações com equipe multiprofissional. Todas as } \\
\text { lactantes estavam amamentando e realizando distanciamento social e cuidados de higiene } \\
\text { pessoal, sendo reforçado pelas consultoras a importância do acesso às fontes confiáveis de } \\
\text { informação. Quanto aos problemas mamários, foram realizadas intervenções preventivas e } \\
\text { curativas. Percebeu-se a satisfação das lactantes pelo serviço. }\end{array}$ \\
\hline
\end{tabular}

\section{Discussão}

A partir da análise dos resultados dos estudos selecionados pode-se observar que não foi identificado no leite materno a presença do vírus SARS-CoV-2, não havendo justificativas para a suspensão da amamentação.
De acordo com a OMS devemos, sempre que possível realizar o contato pele a pele, especialmente após o nascimento, a fim de facilitar a adaptação do RN para o mundo exterior (estabilizando a temperatura do bebê, frequência respiratória, frequência cardíaca e açúcar no sangue) e estabelecer a amamentação ${ }^{8}$. 
Aleitamento materno e a pandemia da COVID-19

Os estudos mostram que para a amamentação ser efetiva, é necessário realizar políticas de promoção de saúde no período pré-natal, e um acompanhamento profissional durante o puerpério sendo necessário uma equipe multiprofissional durante todo o período perinatal , para que após o nascimento aconteça o contato pele a pele e o início da amamentação na primeira hora de vida, bem como para manter o aleitamento exclusivo até os 6 meses e necessário durante a licença maternidade uma rede de apoio para manter o bem-estar materno ${ }^{9}$.

A COVID-19 trouxe consequências para todos, assim como para o binômio mãe e filho, com o distanciamento social e o suporte profissional reduzido, houve mudança no apoio do aleitamento materno ${ }^{9,10}$.

O incentivo ao aleitamento materno, as ações de motivação e esclarecimento sobre a importância do leite para o binômio mãe-bebê, sofreram alterações durante a pandemia, devido à atenção das autoridades de saúde serem totalmente direcionadas ao combate ao novo coronavírus $^{9}$. De acordo com a FEBRASAGO não é recomendado fazer contato pele a pele entre recém-nascido e mãe após o parto. Estudos já demonstraram a presença do RNA da SARS-CoV-2 em amostras de sangue, urina e fezes. Por esse motivo é recomendável a secagem e aquecimento do RN seguidas pelo banho. O clampeamento oportuno do cordão deve-se aguardar de 1 a 3 minutos, uma vez que não haveria maior risco de transmissão vertical, frente aos dados atuais ${ }^{11}$.

Existem diversos associados ao aleitamento materno que repercutem durante toda a vida, muitos estudos apontam que crianças com aleitamento exclusivo diminuem impactos na saúde como alergias, infecções, diarreias, doenças respiratórias, otites, diminui as chances de desenvolver obesidade e diabetes tipo $2^{1,2}$. Sendo assim a amamentação reduz a morbimortalidade infantil, diminuindo os custos com tratamento de doenças nos sistemas de saúde ${ }^{2}$.

No início da pandemia, não se sabia se poderia ser transmitido verticalmente da mãe para o bebê, no útero ou pós-parto, por inalação respiratória direta ou amamentação. Essas preocupações eram transmitidas em noticiários e nas redes sociais, causando medos e angústias para as mulheres; sendo estes, fatores estressantes que podem impactar negativamente na amamentação ${ }^{9,10}$.

Uma pesquisa realizada na China em janeiro/2020 não detectou a presença de SARS-CoV-2 no líquido amniótico, sangue do cordão umbilical, esfregaço da garganta neonatal e nas amostras de leite materno coletadas de seis pacientes ${ }^{12,13}$. Em outro estudo realizado na Espanha em março/abril de 2020, 21 recém-nascidos (RN) foram amamentados e todos tiveram resultados negativos para infecção por SARS-CoV-2, evidenciando a segurança na amamentação ${ }^{14}$.

As informações baseadas em evidências, precisas e de fontes confiáveis que são transmitidas para a população materna são capazes de beneficiá-las se estas forem absorvidas, as informações recentes mostram que não há evidências suficientes sobre a possibilidade de transmissão SARS-CoV-2 através de outros fluidos corporais, como o leite
Simão ALS, Chouzende BO, Duarte KOR, Rodrigues SG, Aver LA, Saco MC materno. Mesmo se a transmissão através da amamentação ocorresse, seria necessário avaliar os riscos de longo prazo associados com a interrupção da amamentação, considerando que a maioria dos casos de SARS-CoV-2 relatados em crianças seguem seu curso com sintomas leves ${ }^{15,16}$.

O leite materno fornece não apenas uma variedade de nutrientes para crescimento e desenvolvimento infantil, mas também muitos componentes bioativos, incluindo anticorpos, para fornecer proteção contra microrganismos patogênicos no início da vida. Os anticorpos circulantes podem entrar no leite materno e ser entregue ao recémnascido, proporcionando-Ihes uma imunização passiva, o que confere proteção contra muitas doenças ${ }^{17,18}$

Desde 18 de março de 2020, a Organização Mundial da Saúde (OMS) recomenda que mulheres com COVID-19 podem amamentar se desejarem fazê-lo, partindo da ideia de que através do leite materno os bebês recebem anticorpos e fatores anti-infecciosos que ajudam a protegêlos contra infecções ${ }^{13}$.

Além de todos os benefícios do aleitamento materno para o RN, analisando o ponto de vista do bemestar emocional materno, estudos comprovam que amamentar facilita o fortalecimento do vínculo mãe-bebê, diminuindo os riscos de a mãe desenvolver uma depressão pós-parto (DPP) ${ }^{13}$. Sendo o leite materno capaz de transferir anticorpos maternos para a criança, protegendo-a de possíveis infecções. ${ }^{6}$

Em uma pesquisa realizada em julho/2020 com mulheres do Reino Unido, mostra que 70,3\% mulheres pararam de amamentar devido à falta de apoio face a face, $20,9 \%$ se preocupam com a segurança da amamentação e 6,5\% interromperam a amamentação devido aos sintomas da COVID- $19^{9}$.

No contexto de uma pandemia, a falta de redes de apoio das mulheres e seus familiares se tornaram um fator preocupante, pois a presença física da família ocupa o primeiro lugar entre as referências das mulheres brasileiras. Ter um familiar possibilita dois aspectos especiais: o cuidado da família com o RN e o apoio à mãe, dando-lhe força e melhorando sua autoestima ${ }^{11}$.

As mulheres, principalmente nas primeiras semanas de amamentação, na qual as necessidades de cuidados são maiores, foram afetadas por não poderem ver os profissionais de saúde pessoalmente ou por sentirem-se desconfortáveis e com medo durante a consulta ${ }^{9,10}$.

Estudo mostra que para algumas mulheres estar mais tempo em casa facilitou claramente a amamentação, apesar de outras sofrerem de ansiedade e estresse devido às responsabilidades com os cuidados com o $\mathrm{RN}^{10}$.

As direções de Sociedade Italiana de Neonatologia (SIN), Sociedade Espanhola de Neonatologia (SeNeo) e União Europeia Neonatal \& Perinatal Societies (UENPS) destacam a importância de manter os cuidados necessários durante o aleitamento materno, como: higiene das mãos antes e depois do contato com o bebê, higiene respiratória e uso de máscara e realizar a desinfecção das superfícies ${ }^{12}$.

Houve mudanças também nos Bancos de Leite Humano (BLH), começaram a usar os meios digitais para 
enviar folhetos de orientação e cuidados, ordenha e doação. Com o distanciamento social, o atendimento presencial foi reduzido, as ações presenciais foram direcionadas à maternidade, aos alojamentos conjuntos e às Unidade de Tratamento Intensivo Neonatal (UTIN). As dúvidas e dificuldades com a amamentação estão sendo sanadas por telefone, chamadas de vídeos e através das mídias sociais ${ }^{19}$.

A Nota Técnica no 5/2020 do Ministério da Saúde (MS), em relação às condutas para a doação de leite materno aos BHLs e Postos de Coleta de Leite Humano (PCLH) no contexto da infecção pelo novo coronavírus, recomenda a doação de leite humano somente por lactantes saudáveis e sem contato domiciliar com pessoa com síndrome gripal. Essa medida faz parte das ações dos BHLs e PCHLs, responsáveis por orientar as candidatas à doação, conforme protocolos do MS e em atenção à RDC n.o 171/2006 ${ }^{19}$.

Pesquisadores relataram que nenhum dos estudos usando detecção de ácido nucléico para o vírus COVID-19 teve validação de sua coleta e métodos analíticos para uso no leite humano, ou descreveu a presença de SARS-CoV-2 viável em amostras ${ }^{20}$.

Sabendo dos benefícios da amamentação, a OMS recomenda fortemente que as mulheres com COVID-19 sejam incentivadas e apoiadas a amamentar, sendo assim, não há recomendação para a suspensão do aleitamento materno na transmissão de vírus respiratório ${ }^{18,20}$.

Em todo o mundo foram criadas medidas que envolvem práticas não farmacológicas individuais básicas para reduzir a transmissão do novo coronavírus, como a higienização das mãos, etiqueta respiratória, uso de máscaras, distanciamento físico, medidas ambientais, entre outras. Se a lactante não se sentir segura para fazer a amamentação enquanto estiver com coronavírus, recomenda-se que seu leite seja retirado e ofertado à criança. Considerando que a mãe infectada pode transmitir o vírus através de gotículas respiratórias, é recomendado tomar as seguintes medidas preventivas para entrar em
Aleitamento materno e a pandemia da COVID-19

Simão ALS, Chouzende BO, Duarte KOR, Rodrigues SG, Aver LA, Saco MC contato com a criança, lavar as mãos por pelo menos 20 segundos antes de tocar o bebê ou antes de retirar o leite materno (extração manual ou na bomba extratora); usar máscara facial (cobrindo completamente nariz e boca) durante as mamadas e evitar falar ou tossir durante a amamentação; a máscara deve ser imediatamente trocada em caso de tosse ou espirro ou a cada nova mamada ${ }^{15,18}$.

De acordo com os estudos existem diversos fatores que impactam no aleitamento materno frente ao SARS-CoV2, podemos citar que a insegurança das lactantes é um grande fator que impede a amamentação e as incertezas que ao longo da pandemia cercam a mesma.

\section{Considerações Finais}

Os benefícios do aleitamento materno de mulheres com COVID-19 são comprovados por estudos realizados a partir de experiências, sendo seguro, à medida que, as mães mantenham as medidas de controle de infecção adequadas para evitar o contágio mãe-bebê. Com isso, é recomendado que as mulheres com COVID-19 sejam orientadas e apoiadas no processo de amamentação.

Observa-se um impacto negativo na vida dessas mulheres e seus bebês, pois quando o aleitamento materno é interrompido, gera consequências emocionais e físicas ao binômio, dos quais podemos citar: aumento risco de depressão puerperal, complicações na mama como ingurgitamento e mastite, risco de maternidade prejudicada devido ao rompimento do vínculo e aumento da suscetibilidade a infecções, relacionado ao neonato. Nota-se durante a busca uma escassa quantidade de estudos relacionados a temática abordada.

A atuação da equipe multiprofissional, tem um importante papel na manutenção do aleitamento, a fim de reduzir danos ao binômio através da realização de atividades na promoção de saúde no período pré-natal, amenizando assim os sentimentos da gestante de ansiedade, medo e tensão frente a pandemia da COVID-19.

\section{Referências}

1. Taveiro EAN, Vianna EYS, Pandolfi MM. Adesão ao Aleitamento Materno Exclusivo em Bebês de 0 a 6 Meses Nascidos em um Hospital e Maternidade do Município de São Paulo. Rev. bras. ciênc. Saúde. 24(1):71-82, [Internet] 2020. [acesso em 09 jan 2021] Disponível em: https://periodicos.ufpb.br/ojs2/index.php/rbcs/article/view/44471/29834

2. Brasil. Ministério da saúde. Aleitamento materno [Internet] 2020. [acesso em 09 jan 2021] Disponível em: https://portalarquivos.saude.gov.br/campanhas/amamentacao/

3. Organização Panamericana de Saúde (OPAS). Organização Mundial da Saúde (OMS). Aleitamento materno nos primeiros anos de vida salvaria mais de 820 mil crianças menores de cinco anos em todo o mundo. [Internet]2018. [acesso em 08 jan 2021] Disponível em: https://www.paho.org/bra/index.php?option=com_content\&view=article\&id=5729:aleitamento-materno-nos-primeiros-anos-de-vidasalvaria-mais-de-820-mil-criancas-menores-de-cinco-anos-em-todo-o-mundo\&Itemid $=820$

4. Ministério da Saúde (BR). Sobre a doença. [Internet] 2020. [acesso em 10 jan 2021] Disponível em: https://coronavirus.saude.gov.br/sobre-a-doenca\#o-que-e-covid

5. Organização Panamericana de Saúde (OPAS). Folha informativa COVID-19. [Internet] 2021. [acesso em 10 jan 2021] Disponível em: https://www.paho.org/bra/index.php?option=com_content\&view=article\&id=6101: covid19\&ltemid=875

6. World Health Organization. Breastfeeding advice during the COVID-19 outbreak. [Internet] 2020. [acesso em 12 jan 2021] Disponível em: http://www.emro.who.int/noncommunicable-diseases/campaigns/breastfeeding-advice-during-the-covid-19-outbreak.html

7. Mendes KDS, Silveira RCCP, Galvão CM. Revisão integrativa: método de pesquisa para a incorporação de evidências na saúde e na enfermagem. Texto Contexto Enferm [Internet]. 2008 Out-Dez; 2017(4): 758-64. [acesso em 12 jan 2021] Disponível em http://www.scielo.br/pdf/tce/v17n4/18.pdf 
Aleitamento materno e a pandemia da COVID-19

Simão ALS, Chouzende BO, Duarte KOR, Rodrigues SG, Aver LA, Saco MC

8. Pereira A, et al. Breastfeeding mothers with COVID-19 infection: a case series. International Breastfeeding Journal, 2020. [acesso em 12 jan. 2021]. Disponível em: https://doi.org/10.1186/s13006-020-00314-8

9. Brown A, Shenker N. Experiences of breastfeeding during COVID19: Lessons for future practical and emotional support. Matern Child Nutr. 2021;17 e13088. [Internet] 2020. [acesso em 10 jan 2021] Disponível em: https://onlinelibrary.wiley.com/doi/epdf/10.1111/mcn.13088

10. Ceulemans M, Verbakel JY, Calsteren K, Eerdekens A, Allegaert K, Foulon V. SARS-CoV-2 Infections and Impact of the COVID-19 Pandemic in Pregnancy and Breastfeeding: Results from an Observational Study in Primary Care in Belgium. Int. J. Environ. Res. Public Health 2020, 17, 6766, 2020.

11. Federação Brasileira das Associações de Ginecologia e Obstetrícia. Protocolo de Atendimento no Parto, Puerpério e Abortamento durante a pandemia da COVID-19. Federação Brasileira das Associações de Ginecologia e Obstetrícia [Internet]. [acesso em 15 jan 2021 ]. Disponivel em: https://www.febrasgo.org.br/pt/covid19/item/1028-protocolo-de-atendimento-no-parto-puerperio-e-abortamentodurante-a-pandemia-da-covid-19

12. Lima ACMACC, Chaves AFL, Oliveira MG, Lima SAFCC, Machado MMT, Oriá MOB. Consultoria em amamentação durante a pandemia COVID-19: relato de experiência. Esc. Anna Nery [Internet]. 2020; Spe. [Acesso em 12 jan 2021]. Disponível em: https://www.scielo.br/scielo.php?script=sci_arttext\&pid=S1414-81452020000500602

13. Wu Y, et al. Coronavirus disease 2019 among pregnant Chinese women: case series data on the safety of vaginal birth and breastfeeding. BJOG: An International Journal of Obstetrics \& Gynaecology. 2020;127(9). [acesso em 12 jan 2021]. Disponível em: https://obgyn.onlinelibrary.wiley.com/doi/10.1111/1471-0528.16276

14. Pereira A, Melguizo SC, Adrien M, Fuentes L, Marin E, Medica TP. Clinical course of coronavirus disease-2019 in pregnancy. Acta Obstetricia et Gynecologica Scandinavica [Internet]. 2020;99(7). [acesso em 12 jan 2021]. Disponível em: https://obgyn.onlinelibrary.wiley.com/doi/full/10.1111/aogs.13921

15. Aquino TF, Teixeira Júnior RM, José ESS, Silva JDD. Pandemia de COVID-19: o olhar da população em relação às medidas preventivas. Glob Acad Nurs. 2020;1(3):e43. [Internet] 2020. [acesso em 19 jan 2021] Disponível em: https://doi.org/10.5935/2675-5602.20200043

16. Gabriel MAM, et al. Negative Transmission of SARS-CoV-2 to Hand-Expressed Colostrum from SARS-CoV-2-Positive Mothers. Breastfeed Med. 2020 Aug;15(8):492-494. [acesso em 12 jun. 2021]. Disponível em: https://doi.org/10.1089/bfm.2020.0183

17. Yu Y, et al. Breastfed 13-month-old infant of a mother with COVID-19 pneumonia: a case report. International Breastfeeding Journal [Internet]. 2020. [acesso em 12 jan. 2021]. Disponível em: https://doi.org/10.1186/s13006-020-00305-9

18. Ministério da Saúde (BR). Secretaria de Atenção primária à saúde. Nota Técnica n.o 09/2020 - DAPES/SAPS/MS [Internet]. [acesso em 12 jan 2021]. Disponível em: https://docs.bvsalud.org/biblioref/2020/05/1096466/notatecnicaamamentacao92020dapessapsms03abr2020covid-19.pdf

19. Marchiori GRS, et al. Ações da enfermagem nos bancos de leite humano em tempos de COVID-19. Rev. Bras. Enferm. vol.73 supl.2 Brasília 2020 Epub 26-Out-2020. [Internet] 2020. [acesso em 12 jan 2021] Disponível em: https://www.scielo.br/scielo.php?script=sci_arttext\&nrm=iso\&lng=pt\&tlng=pt\&pid=S0034-71672020001400155

20. Lebrão CW, et al. Early Identification of IgA Anti-SARSCoV-2 in Milk of Mother With COVID-19 Infection. J Hum Lact. 36(4):609-613, 2020 Nov. [Internet] 2020. [acesso em 12 jan 2021] Disponível em: https://journals.sagepub.com/doi/10.1177/0890334420960433 